\title{
Mobile Robot Localization Using Landmarks
}

\author{
Margrit Betke and Leonid Gurvits
}

\begin{abstract}
We describe an efficient method for localizing a mobile robot in an environment with landmarks. We assume that the robot can identify these landmarks and measure their bearings relative to each other. Given such noisy input, the algorithm estimates the robot's position and orientation with respect to the map of the environment. The algorithm makes efficient use of our representation of the landmarks by complex numbers. The algorithm runs in time linear in the number of landmarks. We present results of simulations and propose how to use our method for robot navigation.
\end{abstract}

Index Terms - Landmark navigation, map algorithms, mobile robot localization, robotics, triangulation.

\section{INTRODUCTION}

W E DESCRIBE AN efficient algorithm for localizing a mobile robot in an environment with landmarks. The robot has sensors that both identify landmarks and measure their bearings relative to each other. Such sensor information is generally uncertain and contains noise. Given the positions of possibly misidentified landmarks on a 2-D map of the environment and noisy measurements of their bearings relative to each other, the algorithm estimates the robot's position with respect to the map of the environment. The algorithm makes efficient use of the geometry of the problem; specifically, the representation of the landmarks by complex numbers. The algorithm runs in time linear in the number of landmarks. Results of simulations are presented that explore the strength of the algorithm.

Why is mobile robot localization important? A robot cannot accurately execute its commands. As a mobile robot moves through its environment, its actual position and orientation always differs from the position and orientation that it is commanded to hold. Wheel slippage is a major source of error. The errors accumulate and the location uncertainty increases over time. Dead-reckoning is not sufficient to locate the robot. Therefore, sensory feedback is needed to locate the robot in its environment.

Consider an autonomous agent, which could be a mobile robot or a human traveler, who uses a map to navigate through an environment that contains landmarks. The landmarks are

Manuscript received June 23, 1994; revised April 27, 1995. The work of M. Betke was supported by NSF Grant ASC-9217041 and Siemens Corp. Research. This paper was recommended for publication by Associate Editor R. Chatila and Editor S. E. Salcudean upon evaluation of the reviewers' comments.

M. Betke was with the Laboratory for Computer Science, Massachusetts Institute of Technology, Cambridge, MA 02139 USA. She is now with the Institute for Advanced Computer Studies, University of Maryland, College Park, MD 20742 USA (e-mail: betke@umiacs.umd.edu).

L. Gurvits is with NEC Research Institute, Princeton, NJ 08540 USA (email: gurvits@ research.nj.nec.com).

Publisher Item Identifier S 1042-296X(97)01034-3. marked on the agent's map. The autonomous agent also has a tool that can measure angles. The agent may use the following algorithm to identify its location in the environment:

1) identify surrounding landmarks in the environment;

2) find the corresponding landmarks on the map;

3) measure the bearings of the landmarks relative to each other;

4) compute your position efficiently.

If the first three steps can be executed without errors three landmarks are sufficient to compute the position of the agent, unless the agent's position and these three landmarks either form a circle, or lie on one line. Then the localization problem does not have a unique solution.

However, in real life an agent makes two kinds of mistakes: 1) some angles are measured with small errors and 2) some landmarks are misidentified. Another source of mistakes could be errors in the map itself. Suppose that the majority of mistakes are of the first type. In this situation we address the following problems:

- estimating the position and orientation of the agent efficiently;

- finding misidentified landmarks and large angle measurement errors.

An algorithm is presented that estimates the agent's position in $O(n)$ operations where $n$ is the number of landmarks on the 2-D map. Large errors due to misidentified landmarks and erroneous angle measurements can be found, discarded and the algorithm can be repeated without them with improved results.

Our work is motivated by a mobile robot called Ratbot that was built at the Learning Systems Department at Siemens Corporate Research. Ratbot is used as a test bed for various machine learning approaches to robot navigation. Ratbot navigates through the corridors of the building at Siemens Corporate Research. It is equipped with a camera that points upwards onto a reflective ball which acts like a mirror of the surroundings (see Fig. 1). The camera setup is due to Judd [1]. Straight lines of objects like picture frames, doors, and walls look like arcs in the images taken by Ratbot's camera. Only a circular, one-dimensional strip of the brightness of each image is analyzed. Landmarks like doors, pictures, and fire extinguishers appear as dark bands on a strip. The strips provide information on the bearing of one landmark relative to another landmark, but not on the distance of the landmarks to the camera (i.e., depth information).

To find the corresponding features of an image that is taken during navigation and an image that is taken in advance and stored in a database, only the strips of the two images need to be compared. Therefore, the correspondence problem, i.e., the problem of identifying features in two images that are the 


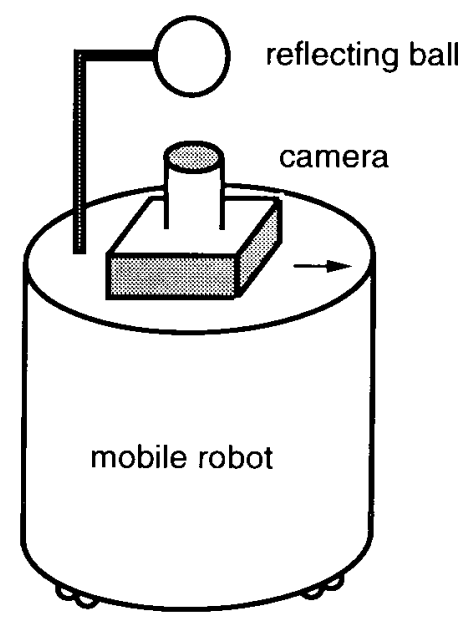

Fig. 1. Illustration of a robot with a camera setup that provides "fish-eyed" circular images of the surroundings.

projections of the same object in the environment, is much easier for pictures taken by Ratbot. Only one-dimensional strips of pixel values of two pictures need to be compared. Hancock and Judd provide an algorithm that finds matching features in two image strips [2].

Once corresponding landmarks are identified, a description of the matching landmarks within the global map of the robot's environment is given. The map of the environment is a twodimensional description of the office building through which the robot navigates. The position of the robot with respect to this map is unknown and needs to be determined.

Our position estimation algorithm has been used by Greiner and Isukapalli [3] to determine which of the landmarks that are visible to Ratbot are useful for its position estimate.

Our localization algorithm is not restricted to robots with a similar camera system as Ratbot's. Our techniques are general and may be used for other camera setups. The algorithm could be used to localize a robot that moves in an assembly plant or to localize an intelligent vehicle in a city.

The paper is organized as follows. Section II describes the work related to our problem. Section III gives a formal description of our problem. Section IV discusses how the robot's position can be estimated using a triangulation approach. Section V describes our position estimation algorithm which uses a different approach that represents landmarks with complex numbers. Section VI proposes how to incorporate our algorithm in an on-line navigation algorithm that estimates the robot's position while the robot is moving.

\section{RELATED WORK}

In recent years several authors have used triangulation approaches for mobile robot localization. Triangulation is a well-known technique for finding a position by means of bearings from fixed points in the environment.

Sugihara [4] addresses a version of the localization problem where the points in the environment look identical (to the contrary, we use landmarks that are distinguishable). The robot measures the direction of rays each of which emanates from the robots position and "pierces" through an observed point.
Sugihara gives an $O\left(n^{3} \lg n\right)$ algorithm for finding the robot's position and orientation such that each ray pierces at least one of the $n$ points in the environment. Avis and Imai [5] and Krotkov [6] extend Sugihara's result to the case that angle measurements include errors.

Sutherland and Thompson [7] address the localization problem using distinguishable landmarks in the environment (as we do). They show that for a given error in angle measurement, the size of the localization error varies depending on the configuration of landmarks.

There is a wide variety of approaches to handle position uncertainties associated with a navigating robot. Some approaches differ from our approach in the way the robot's environment is represented, e.g., Brooks [8], Mataric [9], Elfes [10], and Levitt et al. [11]-[13].

Many authors use a statistical approach for robot navigation, for example, Chatila and Laumond [14], Smith and Cheeseman [15], Kosaka and Kak [16], Crowley [17], Leonard and Durrant-Whyte [18], Watanabe and Yuta [19], Burlina, DeMenthon and Davis [20], and Matthies and Shafer [21].

Approaches to handle uncertainties in a robot's position can also be distinguished by the kind of data available to the robot. Our method uses visual input. Other sensors that have been used for position estimation are sonar sensors [22], odometry [23], GPS [24], and ultrasonic beacons [25].

We address mobile robot localization as a problem of relating robot-centered information with global map information. Relating a camera-centered coordinate system to an external coordinate system is called exterior orientation in the field of photogrammetry (see, for example, [26]). Photogrammetry deals with the problem of how objects in the environment are related to their images in camera systems with different orientations and positions. For robot manipulators, the problem of relating a camera-centered coordinate system to a world-centered coordinate system is called the handeye transformation. The camera information (eye) is used to guide a robot arm or a mobile robot (hand) [27]. Our paper contributes a solution to the problems of exterior orientation and hand-eye transformation.

\section{The Position Estimation Problem}

In this section we define the problem of estimating a robot's position and orientation in its environment given a global map of the environment and bearings of landmarks measured relative to each other at the robot's position.

We call the coordinate system of the map of the environment the external coordinate system. It is spanned by axes $x^{(e)}$ and $y^{(e)}$. We distinguish it from the robot-centered coordinate system spanned by axes $x^{(r)}$ and $y^{(r)}$. A landmark $z$ can be described in both coordinate systems. Vector $z^{(e)}$ describes a landmark in the external coordinate system (see Fig. 2, top). Vector $z^{(r)}$ describes a landmark in the robot-centered coordinate system (see Fig. 2, bottom).

The robot's position is described by vector $p=\left(p_{x}, p_{y}\right)$ in the external coordinate system. Vector $p$ links the origins of both coordinate systems. The robot is oriented in direction of axis $x^{(r)}$. The robot's orientation is described by angle $\theta$ be- 


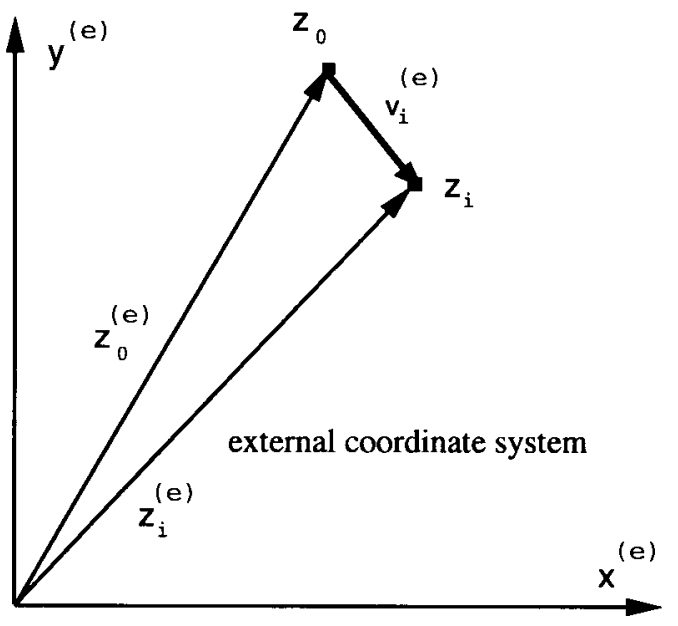

(a)

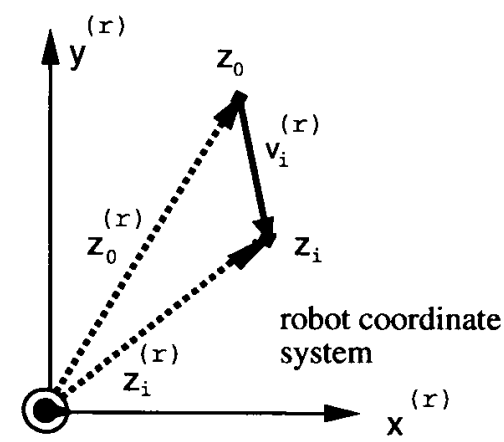

(b)

Fig. 2. (a) Environment with external coordinate system $\left(x^{(e)}, y^{(e)}\right)$. Two landmarks, $z_{0}$ and $z_{i}$, are shown. (b) Environment with robot coordinate system $\left(x^{(r)}, y^{(r)}\right)$. The robot's position is illustrated by a black circled disk. The robot is orientated in the direction of the coordinate axis $x^{(r)}$.

tween the external coordinate axis $x^{(e)}$ and the robot-centered coordinate axis $x^{(r)}$. Fig. 3 illustrates how an external and a robot-centered coordinate system are linked given position vector $p$ and orientation angle $\theta$.

Having a map of the robot's environment means that the vectors $z_{0}^{(e)}, \cdots, z_{n}^{(e)}$ in the external coordinate system are given. The robot measures the bearing of each landmark relative to the direction in which the robot is oriented: Angle $\tau_{i}$ is the angle subtended by landmark vector $z_{i}^{(r)}$ and axis $x^{(r)}$ for $i=0, \cdots, n$

$$
\tau_{i}=\angle\left(z_{i}^{(r)}, x^{(r)}\right.
$$

We call the angle obtained from measuring the bearing of one landmark relative to another landmark the "visual angle" between the two landmarks [7].

The robot does not have a way to measure depth, i.e., it does not know its distance $\left|z_{i}^{(r)}\right|$ to the $i$ th landmark $z_{i}$ for all $i$.

Note that if the orientation angle $\theta$ is zero then we can express the vector between two landmarks $z_{0}$ and $z_{i}$ as vector $v_{i}^{(r)}=z_{0}^{(r)}-z_{i}^{(r)}=v_{i}^{(e)}=z_{0}^{(e)}-z_{i}^{(e)}$ (see Fig. 3).

Now we can formulate our problem: Given the external positions $z_{0}^{(e)}, \cdots, z_{n}^{(e)}$ of $n+1$ landmarks and corresponding angle measurements $\tau_{0}, \cdots, \tau_{n}$, estimate the position $p$ and the orientation $\theta$ of the robot in the environment.

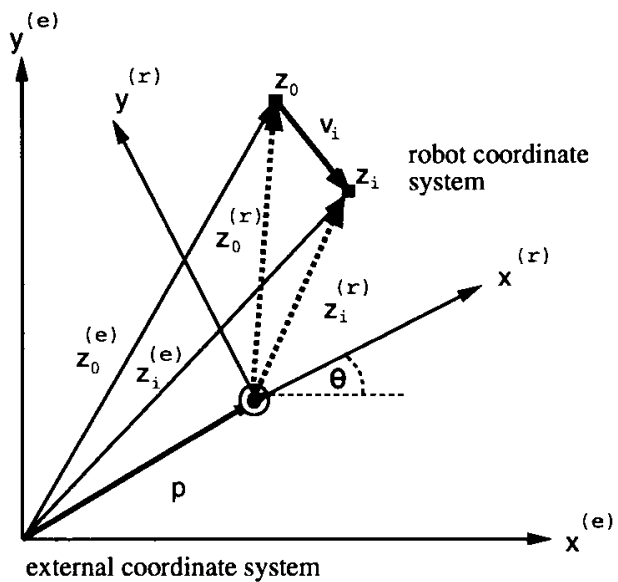

Fig. 3. Environment with external and robot coordinate system: Both vector $z_{i}^{(e)}$ in the external coordinate system and vector $z_{i}^{(r)}$ in the robot coordinate system point to landmark $z_{i}$. The robot's location is defined by position vector $p$ and orientation angle $\theta$.

\section{A TRIANGULATION APPROACH}

This section shows how to apply well-known triangulation techniques to the robot localization problem. First we consider the localization problem for perfect data, then for noisy data.

\section{A. Triangulation with Perfect Data}

If the visual angle $\varphi$ between two landmarks $z_{0}$ and $z_{1}$ measured at an unknown position $p$ and the distance between the landmarks is known, then position $p$ lies on an arc of a circle spanned by landmarks $z_{0}$ and $z_{1}$ [28] (see Fig. 4(a)).

A third landmark $z_{2}$ is needed to determine position $p$ uniquely: it is the intersection of the circle through landmarks $z_{0}$ and $z_{1}$ and the circle through landmarks $z_{1}$ and $z_{2}$ (see Fig. 4(b)). Note that $p$ cannot be determined uniquely if landmarks $z_{0}, z_{1}$ and $z_{2}$ lie on a circle.

One approach to compute position $p$ is to describe the two circles analytically and determine their intersection. The law of cosine can be used to compute the radius of each circle given the visual angles and distances between the landmarks. The coordinates of the center of the circles can then be computed from the coordinates of landmarks $z_{0}, z_{1}$ and $z_{2}$. Once vector $p$ is known, the orientation of the robot, i.e., the direction of $x^{(r)}$ is known.

Another way of triangulating position $p$ is to determine the distance between the robot and the three landmarks. We follow this approach in the next section.

\section{B. Triangulation with Noisy Data}

The triangulation method described above does not compute the exact position if the input data is noisy. Using several landmarks yields a more robust position estimate. Given $n$ landmarks, there are $\left(\begin{array}{l}n \\ 3\end{array}\right)$ combinations of three landmarks that can be used to compute $\left(\begin{array}{l}n \\ 3\end{array}\right)$ position estimates by the triangulation method described above. One way of combining these estimates to obtain a final position estimate is to compute their average.

In the following we outline a different triangulation method that uses $n$ landmarks and is based on estimating the distance 


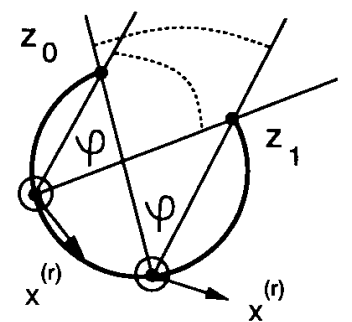

(a)

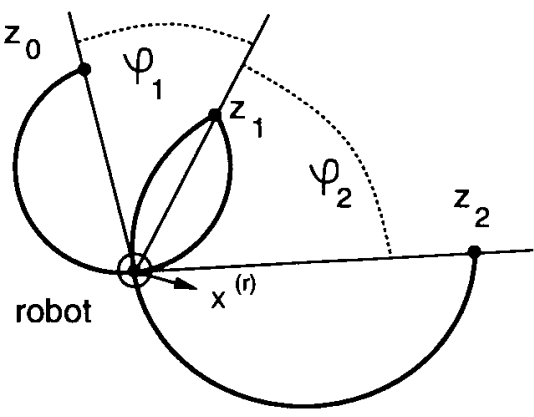

(b)

Fig. 4. Triangulation with perfect data: (a) Scenario with two landmarks: The robot's position is somewhere on the arc shown. The robot is oriented in the direction of $x^{(r)}$. (b) Scenario with three landmarks: The robot's position is at the point of intersection of the two arcs shown. The robot's orientation can be uniquely determined from $x^{(r)}$.

between the robot and the landmarks. (Note that this is not the method that we propose.)

Applying the law of cosine, the relationship between two landmarks $z_{i}$ and $z_{j}$ can be written as

$$
\left|v_{i j}\right|^{2}=\left|z_{i}^{(r)}\right|^{2}+\left|z_{j}^{(r)}\right|^{2}-2\left|z_{i}^{(r)}\right|\left|z_{j}^{(r)}\right| \cos \varphi_{i j}
$$

where $\left|v_{i j}\right|$ is the (known) distance between $z_{i}$ and $z_{j}, \varphi_{i j}$ is the visual angle between $z_{i}$ and $z_{j}$ at the robot's position, and $\left|z_{i}^{(r)}\right|$ and $\left|z_{j}^{(r)}\right|$ are the unknown distances to the landmarks (see Fig. 5). Angle $\varphi_{i j}$ is computed from measured angles $\tau_{i}$ and $\tau_{j}: \varphi_{i j}=\tau_{i}-\tau_{j}$.

We can apply the law of cosine to all possible sets of two landmarks and get a system of equations which is overdetermined and can be solved using a least squares approach. Once the lengths $\left|z_{i}^{(r)}\right|, \cdots,\left|z_{n}^{(r)}\right|$ are found, another overdetermined set of equations needs to be solved to find the position $p=\left(p_{x}, p_{y}\right)$ of the robot: $\left|z_{i}^{(r)}\right|^{2}=\left(x_{i}-p_{x}\right)^{2}+\left(y_{i}-p_{y}\right)^{2}$ for $i=0, \cdots, n$ where $x_{i}$ and $y_{i}$ are the coordinates of $z_{i}^{(r)}$. Once vector $p$ is estimated, we can get an estimate for the orientation angle $\theta$ by computing vector $z_{i}^{(r)}=z_{i}^{(e)}-p$ for some $i$ and then $\theta=\angle\left(z_{i}^{(r)}, x^{(e)}\right)-\tau_{i}$.

In the following we illustrate the method with three landmarks $z_{0}, z_{i}$ and $z_{j}$ (see also Fig. 5). The law of cosine gives us

$$
\begin{aligned}
& \left|v_{0 i}\right|^{2}=\left|z_{0}^{(r)}\right|^{2}+\left|z_{i}^{(r)}\right|^{2}-2\left|z_{0}^{(r)}\right|\left|z_{i}^{(r)}\right| \cos \varphi_{i 0} \\
& \left|v_{0 j}\right|^{2}=\left|z_{0}^{(r)}\right|^{2}+\left|z_{j}^{(r)}\right|^{2}-2\left|z_{0}^{(r)}\right|\left|z_{j}^{(r)}\right| \cos \varphi_{j 0} \\
& \left|v_{i j}\right|^{2}=\left|z_{i}^{(r)}\right|^{2}+\left|z_{j}^{(r)}\right|^{2}-2\left|z_{i}^{(r)}\right|\left|z_{j}^{(r)}\right| \cos \varphi_{i j} .
\end{aligned}
$$

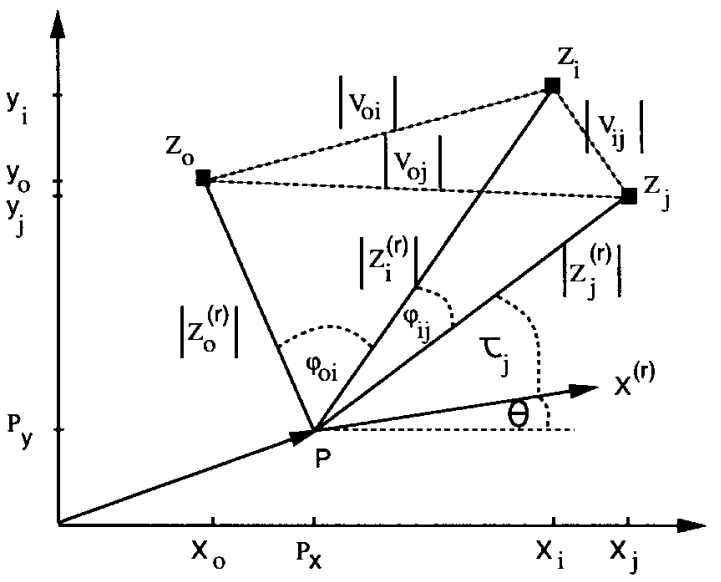

Fig. 5. Position estimation using the law of cosine.

First we could solve this system of nonlinear equations using a least squares method. (A review of the least squares method for solving linear equations is given in Section $\mathrm{V}$-A.) Then we could express the now known lengths $\left|z_{0}^{(r)}\right|,\left|z_{i}^{(r)}\right|$ and $\left|z_{j}^{(r)}\right|$ by the (unknown) coordinates of the position $p=\left(p_{x}, p_{y}\right)$ of the robot and the coordinates $(x, y)$ of the landmarks

$$
\begin{aligned}
& \left|z_{0}^{(r)}\right|^{2}=\left(x_{0}-p_{x}\right)^{2}+\left(y_{0}-p_{y}\right)^{2} \\
& \left|z_{i}^{(r)}\right|^{2}=\left(x_{i}-p_{x}\right)^{2}+\left(y_{i}-p_{y}\right)^{2} \\
& \left|z_{j}^{(r)}\right|^{2}=\left(x_{j}-p_{x}\right)^{2}+\left(y_{j}-p_{y}\right)^{2} .
\end{aligned}
$$

Subtracting the second and third equation from the first we get two equations linear in the unknowns $p_{x}$ and $p_{y}$ :

$$
\begin{aligned}
\left|z_{0}^{(r)}\right|^{2}-\left|z_{i}^{(r)}\right|^{2}= & x_{0}^{2}-x_{i}^{2}+2 p_{x}\left(x_{i}-x_{0}\right)+y_{0}^{2}-y_{i}^{2} \\
& +2 p_{y}\left(y_{i}-y_{0}\right) \\
\left|z_{0}^{(r)}\right|^{2}-\left|z_{j}^{(r)}\right|^{2}= & x_{0}^{2}-x_{j}^{2}+2 p_{x}\left(x_{j}-x_{0}\right)+y_{0}^{2}-y_{j}^{2} \\
& +2 p_{y}\left(y_{j}-y_{0}\right) .
\end{aligned}
$$

Finally, we can solve for $p_{x}$ and $p_{y}$ and obtain an estimate for the position $p=\left(p_{x}, p_{y}\right)$.

Triangulation with noisy data as described above is based on solving nonlinear equations with complicated closed-form solutions. However, standard algorithms that provide leastsquares solutions for large numbers of nonlinear equations take too long for real-time robot navigation. In the following section, we introduce a new linear-time approach to localizing a mobile robot which is based on a least-squares solution of a linear set of equations.

\section{LINEAR POSITION ESTIMATION}

This section describes an efficient localization algorithm that runs in time linear in the number of landmarks. Instead of using the triangulation method described above, we use a method based on the complex number representation of the landmarks. This representation is the key idea to get a set of linear equations whose solution is a set of position estimates. (Triangulation methods may also provide a set of position estimates but as a solution to nonlinear equations.) We describe 
this set of $m$ linear equations as a vector equation. To solve this particular vector equation, we do not need an $O\left(\mathrm{~m}^{3}\right)$ matrix inversion algorithm. Instead we introduce an $O(m)$ algorithm which takes advantage of the special structure of the matrix and the right side of the vector equation. In the following, we describe how to set up and solve this vector equation.

A landmark $z$ can be written as a complex number in the robot-centered coordinate system. For each landmark $z_{i}^{(r)}$ we have an expression

$$
z_{i}^{(r)}=l_{i} e^{j \tau_{i}} \quad \text { for } i=1, \cdots, n
$$

where length $l_{i}$ is the unknown distance of the robot to landmark $z_{i}^{(r)}$, angle $\tau_{i}$ is the measured angle between $z_{i}^{(r)}$ and the coordinate axis $x^{(r)}$, and $j=\sqrt{-1}$.

We pick a reference landmark $z_{0}^{(r)}$ and compute the angles $\varphi_{i}=\tau_{i}-\tau_{0}$ for $i=1, \cdots, n$. (Landmark $z_{0}^{(r)}$ may be chosen to be the most reliable landmark if such reliability information is available.) Dividing the complex number representation of landmarks $z_{1}^{(r)}, \cdots, z_{n}^{(r)}$ by $z_{0}^{(r)}$ for $i=1, \cdots, n$ yields a set of equations that includes the visual angles $\varphi_{i}$ between landmark $z_{i}$ and landmark $z_{0}$

$$
\frac{z_{i}^{(r)}}{z_{0}^{(r)}}=\frac{l_{i}}{l_{0}} e^{j\left(\tau_{i}-\tau_{0}\right)}=\frac{l_{i}}{l_{0}} e^{j \varphi_{i}} .
$$

Given that $z_{i}^{(r)}=z_{0}^{(r)}+v_{i}^{(r)}$, (1) becomes

$$
\frac{z_{0}^{(r)}+v_{i}^{(r)}}{z_{0}^{(r)}}=\frac{l_{i}}{l_{0}} e^{j \varphi_{i}} \quad \text { for } i=1, \cdots, n .
$$

After some algebra we obtain a set of equations whose unknowns are vectors $z_{0}^{(r)}$ and $v_{i}^{(r)}$ and ratios $l_{i} / l_{0}$, for $i=1, \cdots, n$

$$
\frac{1}{z_{0}^{(r)}}=\frac{l_{i}}{l_{0}} \frac{1}{v_{i}^{(r)}} e^{j \varphi_{i}}-\frac{1}{v_{i}^{(r)}} \quad \text { for } i=1, \cdots, n
$$

To remove the dependence on $z_{0}^{(r)}$, we substitute the left-hand side of (2) with the expression on the right-hand side for a different index $k$

$$
\frac{l_{k}}{l_{0}} \frac{1}{v_{k}^{(r)}} e^{j \varphi_{k}}-\frac{1}{v_{k}^{(r)}}=\frac{l_{i}}{l_{0}} \frac{1}{v_{i}^{(r)}} e^{j \varphi_{i}}-\frac{1}{v_{i}^{(r)}}
$$

for $i, k=1, \cdots, n$ and $k \neq i$. The only unknowns in (3) are vectors $v_{i}^{(r)}$ and ratios $l_{i} / l_{0}$. Since the angles $\varphi_{1}, \cdots, \varphi_{n}$ in (3) are independent of the orientation of the robot-centered coordinate system, we can rewrite (3) for a robot-centered coordinate system with a different orientation

$$
r_{k} \frac{1}{\hat{v}_{k}^{(r)}} e^{j \varphi_{k}}-\frac{1}{\hat{v}_{k}^{(r)}}=r_{i} \frac{1}{\hat{v}_{i}^{(r)}} e^{j \varphi_{i}}-\frac{1}{\hat{v}_{i}^{(r)}}
$$

for $i, k=1, \cdots, n$ and $k \neq i$, where landmark $z_{i}$ is described by vector $\hat{z}_{i}^{(r)}, r_{i}=\left|\hat{z}_{i}^{(r)}\right| /\left|\hat{z}_{0}^{(r)}\right|$ and $\hat{v}_{i}^{(r)}=\hat{z}_{i}^{(r)}-\hat{z}_{0}^{(r)}$. We

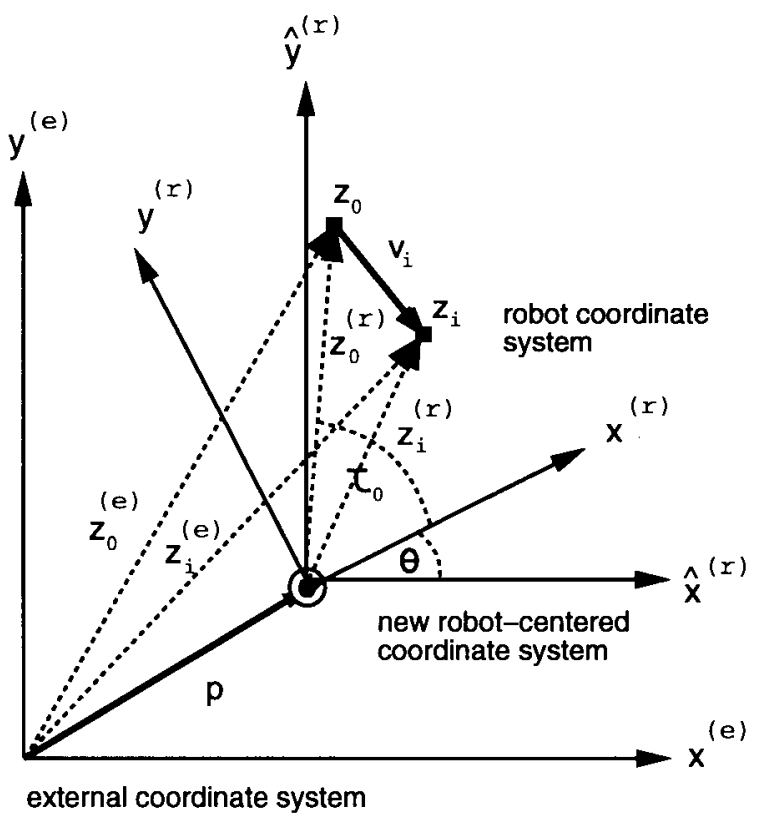

Fig. 6. External coordinate system spanned by $x^{(e)}$ and $y^{(e)}$, robot-centered coordinate system spanned by $x^{(r)}$ and $y^{(r)}$ and robot-centered coordinate system spanned by $\hat{x}^{(r)}$ and $\hat{y}^{(r)}$. The robot is facing in direction of axis $x^{(r)}$.

choose the robot-centered coordinate system that is oriented the same way as the external coordinate system. Thus, its axes $\hat{x}^{(r)}$ and $\hat{y}^{(r)}$ are parallel to axes $x^{(e)}$ and $y^{(e)}$, respectively (see Fig. 6). We pick this particular coordinate system, because we can then write

$$
\hat{v}_{i}^{(r)}=v_{i}^{(e)}=z_{i}^{(e)}-z_{0}^{(e)}
$$

for $i=1, \cdots, n$ which yields equations

$$
r_{k} \frac{1}{v_{k}^{(e)}} e^{j \varphi_{k}}-\frac{1}{v_{k}^{(e)}}=r_{i} \frac{1}{v_{i}^{(e)}} e^{j \varphi_{i}}-\frac{1}{v_{i}^{(e)}}
$$

for $i, k=1, \cdots, n$ and $k \neq i$, whose only unknowns are ratios $r_{1}, \cdots, r_{n}$.

Note that using the robot-centered coordinate system that is spanned by axes $\hat{x}^{(r)}$ and $\hat{y}^{(r)}$ does not imply that the robot knows its orientation in the environment a priori. It does not know its orientation angle $\theta=\angle\left(x^{(r)}, x^{(e)}\right)=\angle\left(x^{(r)}, \hat{x}^{(r)}\right)$. Instead, once (5) are solved we can compute vector $\hat{z}_{0}^{(r)}$ which can then be used to compute the robot's orientation

$$
\theta=\angle\left(\hat{z}_{0}^{(r)}, \hat{x}^{(r)}\right)-\tau_{0}
$$

as illustrated in Fig. 6.

The set of (5) can be transformed into a matrix equation $A r=c$ where $r, c$, and $A$ can be defined as follows: Vector $r=$ $\left(r_{1}, \cdots, r_{n}\right)$ is the vector of the unknown ratios of the length of vectors $\hat{z}_{1}^{(r)}, \cdots, \hat{z}_{n}^{(r)}$. Vector $c$ is a $n(n-1)$ dimensional vector of differences of complex numbers $c_{i}=\left(c_{x i}, c_{y i}\right)=1 / \hat{v}_{i}^{(r)}$ and matrix $A$ is a $n(n-1) \times n$ matrix consisting of complex 
numbers $b_{i}=\left(b_{x i}, b_{y i}\right)=c_{i} e^{j \varphi_{i}}$

$$
A=\left(\begin{array}{ccccccc}
b_{1} & -b_{2} & 0 & 0 & \cdots & 0 & 0 \\
b_{1} & 0 & -b_{3} & 0 & \cdots & 0 & 0 \\
b_{1} & 0 & 0 & -b_{4} & \cdots & 0 & 0 \\
\vdots & & & & & & \\
b_{1} & 0 & 0 & 0 & \cdots & 0 & -b_{n} \\
-b_{1} & b_{2} & 0 & 0 & \cdots & 0 & 0 \\
0 & b_{2} & -b_{3} & 0 & \cdots & 0 & 0 \\
0 & b_{2} & 0 & -b_{4} & \cdots & 0 & 0 \\
\vdots & & & & & & \\
0 & b_{2} & 0 & 0 & \cdots & 0 & -b_{n} \\
\vdots & & & & & & \\
-b_{1} & 0 & 0 & 0 & \cdots & 0 & b_{n} \\
0 & -b_{2} & 0 & 0 & \cdots & 0 & b_{n} \\
0 & 0 & -b_{3} & 0 & \cdots & 0 & b_{n} \\
\vdots & & & & & & \\
0 & 0 & 0 & 0 & \cdots & -b_{n-1} & b_{n}
\end{array}\right)
$$

and

$$
\left(\begin{array}{c}
c_{1}-c_{2} \\
c_{1}-c_{3} \\
c_{1}-c_{4} \\
\vdots \\
c_{1}-c_{n} \\
c_{2}-c_{1} \\
c_{2}-c_{3} \\
c_{2}-c_{4} \\
\vdots \\
c_{2}-c_{n} \\
\vdots \\
c_{n}-c_{1} \\
c_{n}-c_{2} \\
c_{n}-c_{3} \\
\vdots \\
c_{n}-c_{n-1}
\end{array}\right)
$$

A solution of equation $A r=c$ yields a vector $r$ whose components $r_{i}$ can be used to solve equation

$$
\frac{1}{\hat{z}_{0}^{(r)}}=r_{i} \frac{1}{v_{i}^{(e)}} e^{j \varphi_{i}}-\frac{1}{v_{i}^{(e)}}
$$

for some $i$ to obtain vector $\hat{z}_{0}^{(r)}$. (This equation corresponds to (2) and describes the vectors with respect to the robot-centered coordinate system spanned by axes $\hat{x}^{(r)}$ and $\hat{y}^{(r)}$.) Once $\hat{z}_{0}^{(r)}$ is known, the robot position $p$ is

$$
p=z_{0}^{(e)}-\hat{z}_{0}^{(r)}
$$

Note that the system of linear equations $A r=c$ is overdetermined. We have $n$ unknowns $r_{1}, \cdots, r_{n}$ and $n(n-1)$ equations. These equations may be inconsistent, since we have measurement errors. Therefore, we use a "least squares method" to find a solution for which the average error in the $n(n-1)$ equations is minimized.

\section{A. Least Squares Errors and the Pseudo-Inverse}

The "least squares method" is a standard method for solving overdetermined systems of equations [29]. In the following we rederive this method for our particular overdetermined system of equations.

To solve the matrix equation $A r=c$ we choose ratio vector $r$ so as to minimize the "average" error in the $n(n-1)$ equations. We define the average error by the sum of squares

$$
E^{2}=\|A r-c\|^{2} \text {. }
$$

If there is an exact solution to $A r=c$, the error is $E=0$. In practice it is unlikely that there is no error, therefore, we solve the minimization problem

$$
\min \left\{r: E^{2}\right\}=\min \left\{r:\|A r-c\|^{2}\right\} .
$$

The necessary condition of the squared error $E^{2}$ to be minimal is that its derivative with respect to vector $r$ must be zero. The derivative is

$$
\begin{aligned}
\frac{d}{d r} E^{2} & =\frac{d}{d r}\|A r-c\|^{2} \\
& =\frac{d}{d r}\left(r^{T} A^{T} A r-r^{T} A^{T} c-c^{T} A r+c^{T} c\right) \\
& =2 A^{T} A r-2 A^{T} c
\end{aligned}
$$

where $A^{T}$ is the conjugate transpose of $A$. Setting the derivative to zero gives us

$$
\begin{aligned}
& 0=2 A^{T} A r-2 A^{T} c \\
& r=\left(A^{T} A\right)^{-1} A^{T} c
\end{aligned}
$$

provided matrix $A^{T} A$ is nonsingular. In Section $\mathrm{V}-\mathrm{C}$ we give the necessary and sufficient conditions for $A^{T} A$ being nonsingular and argue that they will almost always be fulfilled in practice.

Matrix $A^{+}=\left(A^{T} A\right)^{-1} A^{T}$ is often called the pseudoinverse of matrix $A$ (see for example [30], [29]). In the following section we describe an algorithm called Position Estimator which efficiently calculates vector $r=A^{+} c=$ $\left(A^{T} A\right)^{-1} A^{T} c$ such that the average error in the equations is minimized.

\section{B. The Position Estimation Algorithm}

In this section we describe procedure Position Estimator that estimates the position and the orientation of the robot. In the description of the algorithm we use two kinds of multiplications of complex numbers $a=(\operatorname{Re}(a), \operatorname{Im}(a))$ and $b=(\operatorname{Re}(b), \operatorname{Im}(b))$ : the standard complex multiplication

$a b=(\operatorname{Re}(a) \operatorname{Re}(b)-\operatorname{Im}(a) \operatorname{Im}(b), \operatorname{Re}(a) \operatorname{Im}(b)+\operatorname{Re}(b) \operatorname{Im}(a))$

and the inner product

$$
\bar{a}^{T} b=\operatorname{Re}(a) \operatorname{Re}(b)+\operatorname{Im}(a) \operatorname{Im}(b)
$$

where $\bar{a}=(\operatorname{Re}(a),-\operatorname{Im}(a))$ is the conjugate of $a$. For notational convenience we write the inner product of complex numbers $a$ and $b$ as $a^{T} b$. 


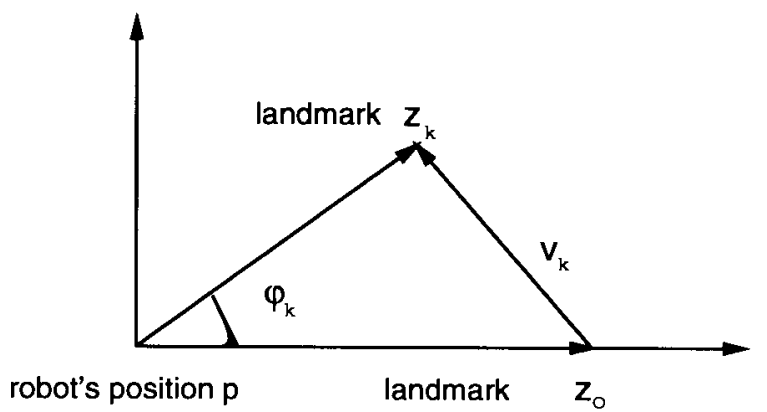

Fig. 7. Robot coordinate system defined as a $z$-plane in the proof of Lemma 3.

Procedure Position Estimator takes as an input the positions of the landmarks $z_{0}^{(e)}, \cdots, z_{n}^{(e)}$ (as given in the external coordinate system) and the angles $\varphi_{1}, \cdots, \varphi_{n}$.

Position Estimator $\left(z_{0}^{(e)}, \cdots, z_{n}^{(e)}, \varphi_{1}, \cdots \varphi_{n}\right)$

1. sum-of- $c_{i}=0$

2. for $i=1$ to $n \quad$ (initialization)

3. $\quad v_{i}=z_{0}^{(e)}-z_{i}^{(e)}$

4. $\quad c_{i}=1 / v_{i}$

5. sum-of- $c_{i}=$ sum-of- $c_{i}+c_{i} \quad$ (calculates $\left.\sum_{i=1}^{n} c_{i}\right)$

6. $b_{i}=c_{i} e^{j \varphi_{i}}$

7. for $i=1$ to $n \quad$ (calculates $\frac{1}{2} A^{T} c$ )

8. $s_{i}=n b_{i}^{T} c_{i}-b_{i}^{T}$ sum-of- $c_{i}$

9. Ratios-Calculator $\left(b_{1}, \cdots, b_{n}, s_{1}, \cdots, s_{n}\right.$ ) (returns $r$ )

10. for $i=1$ to $n$

11. $\hat{z}_{0, i}^{(r)}=1 /\left(r_{i} b_{i}-c_{i}\right)$

12. $p_{i}=z_{0}^{(e)}-\hat{z}_{0, i}^{(r)}$

13. $p=\frac{1}{n} \sum_{i=1}^{n} p_{i} \quad($ robot position $p)$

14. $\theta=\angle\left(\hat{z}_{0}^{(r)}, \hat{x}^{(r)}\right)-\tau_{0}$

After the initialization of vectors $v, c, b$ and $\sum_{i=1}^{n} c_{i}$ in lines 2-6, Position Estimator first computes vector $s=\frac{1}{2} A^{T} C$ in lines 7 and 8 as follows: For each component of vector $s=\frac{1}{2} A^{T} c$ the procedure computes

$$
\begin{aligned}
s_{i} & =(n-1) b_{i}^{T} c_{i}-\sum_{j, j \neq i} b_{i}^{T} c_{j} \\
& =n b_{i}^{T} c_{i}-\sum_{j=1}^{n} b_{i}^{T} c_{j} \\
& =n b_{i}^{T} c_{i}-b_{i}^{T} \sum_{j=1}^{n} c_{j} \\
& =n\left(b_{x i} c_{x i}+b_{x i} c_{x i}\right)-\left(b_{x i} \sum_{j=1}^{n} c_{x j}+b_{y i} \sum_{j=1}^{n} c_{y j}\right)
\end{aligned}
$$

Procedure Position Estimator then determines $r=$ $2\left(A^{T} A\right)^{-1} s$ by calling procedure Ratios-Calculator in line 9 . It next calculates $n$ position estimates $p_{1}, \cdots, p_{n}$ (lines 10-12).

In line 11 of procedure Position Estimator, a set of $n$ solutions $\hat{z}_{0,1}^{(r)}, \cdots, \hat{z}_{0, n}^{(r)}$ for the position of landmark $\hat{z}_{0}^{(r)}$ in the robot-centered coordinate system is calculated using (7)

$$
\hat{z}_{0, i}^{(r)}=\frac{1}{r_{i} b_{i}-c_{i}} \quad \text { for } i=1, \cdots, n
$$

In line 13 of procedure Position Estimator, a set of estimates $p_{1}, \cdots, p_{n}$ for the robot position is calculated using the solutions for the position of landmark $z_{0}$.

If there is no noise in the measurements, the vectors $\hat{z}_{0, i}^{(r)}$ are the same for all indexes $i$. In practice there will be noise, so we take the centroid

$$
p=\frac{1}{n} \sum_{i=1}^{n} p_{i}
$$

of the position estimates $p_{1}, \cdots, p_{n}$ as an average to obtain an estimate of the position $p$ of the robot (line 13 of procedure Position Estimator). In line 14 the orientation angle is computed using (6). (Note that taking the centroid may not be the best way to average the $n$ estimates. We are currently working on determining the best averaging coefficients [31]. They may not be the same for the $x$ - and $y$-coordinates of $p_{i}$.).

Procedure Ratios-Calculator takes as an input the vectors $b$ and $s$ and returns the vector $r=2\left(A^{T} A\right)^{-1} s$. First RatiosCalculator calculates vectors $b_{x}=\left(b_{x 1}, \cdots, b_{x n}\right)$ and $b_{y}=$ $\left(b_{y 1}, \cdots, b_{y n}\right)$ where $b_{x i}$ is the real coordinate of $b_{i}$ and $b_{y i}$ is the imaginary coordinate of $b_{i}$. Then it exploits the special form of matrix $A^{T} A$

$A^{T} A=$

$$
2\left(\begin{array}{ccccc}
(n-1) b_{1}^{T} b_{1} & -b_{1}^{T} b_{2} & -b_{1}^{T} b_{3} & \cdots & -b_{1}^{T} b_{n} \\
-b_{2}^{T} b_{1} & (n-1) b_{2}^{T} b_{2} & -b_{2}^{T} b_{3} & \cdots & -b_{2}^{T} b_{n} \\
\vdots & & & & \\
-b_{n}^{T} b_{1} & -b_{n}^{T} b_{2} & -b_{n}^{T} b_{3} & \cdots & (n-1) b_{n}^{T} b_{n}
\end{array}\right)
$$

Instead of inverting the matrix $A^{T} A$ directly, we write matrix $A^{T} A$ as a sum of a diagonal matrix and two matrices that are outer products (or Grammian matrices, see [30]). In this special form, the matrix $A^{T} A$ can be inverted efficiently

$$
A^{T} A=2\left(n D-b_{x} b_{x}^{T}-b_{y} b_{y}^{T}\right)
$$

where $D$ is a diagonal matrix whose $i$ th entry is $b_{i}^{T} b_{i}=$ $b_{x i}^{2}+b_{y i}^{2}$, and the outer product $b_{x} b_{x}^{T}$ is defined as follows:

$$
b_{x} b_{x}^{T}=\left(\begin{array}{cccc}
b_{x 1}^{2} & b_{x 1} b_{x 2} & \cdots & b_{x 1} b_{x n} \\
b_{x 2} b_{x 1} & b_{x 2}^{2} & \cdots & b_{x 2} b_{x n} \\
& \vdots & & \\
b_{x n} b_{x 1} & b_{x n} b_{x 2} & \cdots & b_{x n}^{2}
\end{array}\right)
$$

Matrix $b_{y} b_{y}^{T}$ can be written analogously.

Then $r=\left(A^{T} A\right)^{-1} A^{T} c=\left(A^{T} A\right)^{-1} 2 s=1 / 2(n D-$ $\left.b_{x} b_{x}^{T}-b_{y} b_{y}^{T}\right)^{-1} 2 s=\left(n D-b_{x} b_{x}^{T}-b_{y} b_{y}^{T}\right)^{-1} s$. In this form $r$ can be calculated (without inverting $n D-b_{x} b_{x}^{T}-b_{y} b_{y}^{T}$ directly) using the following well-known lemma [30]:

Lemma 1: If matrix $K$ is nonsingular and $\left(K^{-1} h\right)^{T} h \neq 1$, then

$$
\left(K-h h^{T}\right)^{-1}=K^{-1}+\frac{K^{-1} h h^{T} K^{-1}}{1-\left(K^{-1} h\right)^{T} h}
$$

Proof: The proof follows directly by verifying equation

$$
I=\left(K-h h^{T}\right)\left(K^{-1}+\frac{K^{-1} h h^{T} K^{-1}}{1-\left(K^{-1} h\right)^{T} h}\right)
$$

where $I$ is the identity matrix.

We apply the formula given in Lemma 1 twice to invert matrix $A^{T} A$. (We show in Section $\mathrm{V}-\mathrm{C}$ that the requirements 


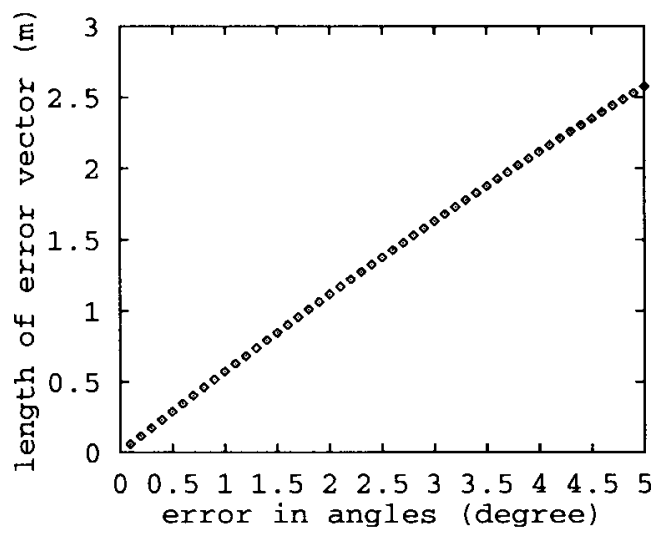

(a)

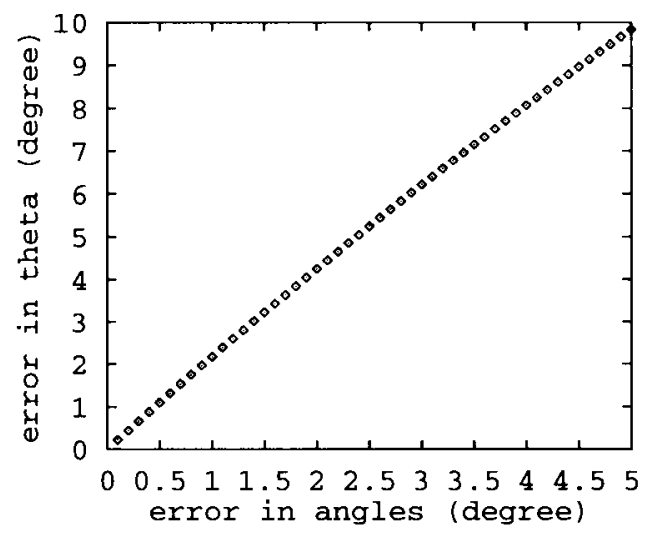

(b)

Fig. 8. Simulation results using algorithm Position Estimator on an input of 11 landmarks and angle measurements with constant error. The error $\left\|p_{\text {actual }}-p\right\|$ in meters (top) and the error $\left|\theta_{\text {actual }}-\theta\right|$ in degrees (bottom) are shown as functions of $\Delta \varphi$ in degrees.

of Lemma 1, i.e., matrix $K$ is nonsingular and $\left(K^{-1} h\right)^{T} h \neq 1$, are indeed met.)

Since $r=\left(A^{T} A\right)^{-1} A^{T} c=\left(\left(n D-b_{x} b_{x}^{T}\right)-b_{y} b_{y}^{T}\right)^{-1} s$, we can apply Lemma 1 to $K=\left(n D-b_{x} b_{x}^{T}\right)$ and $h=b_{y}$ and get

$$
\begin{aligned}
r & =\left(\left(n D-b_{x} b_{x}^{T}\right)-b_{y} b_{y}^{T}\right)^{-1} s \\
& =K^{-1} s+\frac{K^{-1} b_{y} b_{y}^{T} K^{-1}}{1-\left(K^{-1} b_{y}\right)^{T} b_{y}} s \\
& =K^{-1} s+\frac{\left(K^{-1} b_{y}\right)\left(\left(K^{-1} b_{y}\right)^{T} s\right)}{1-\left(K^{-1} b_{y}\right)^{T} b_{y}} .
\end{aligned}
$$

Applying Lemma 1 again, the first term $K^{-1} s$ can be expressed as

$$
\begin{aligned}
K^{-1} s & =\left(n D-b_{x} b_{x}^{T}\right)^{-1} s \\
& =(n D)^{-1} s+\frac{(n D)^{-1} b_{x}\left((n D)^{-1} b_{x}\right)^{T} s}{1-\left((n D)^{-1} b_{x}\right)^{T} b_{x}} .
\end{aligned}
$$

Vector $K^{-1} b_{y}=\left(n D-b_{x} b_{x}^{T}\right)^{-1} b_{y}$ in (9) is calculated by applying the formula given in Lemma 1 which yields

$$
K^{-1} b_{y}=(n D)^{-1} b_{y}+\frac{(n D)^{-1} b_{x}\left((n D)^{-1} b_{x}\right)^{T} b_{y}}{1-\left((n D)^{-1} b_{x}\right)^{T} b_{x}} \text {. }
$$

The inverse of a diagonal matrix is a matrix whose entries on the diagonal are inverted. Thus, the $i$ th diagonal entry of $(n D)^{-1}$ is $1 /\left(n b_{i}^{T} b_{i}\right)=1 /\left(n\left(b_{x i}^{2}+b_{y i}^{2}\right)\right)$.

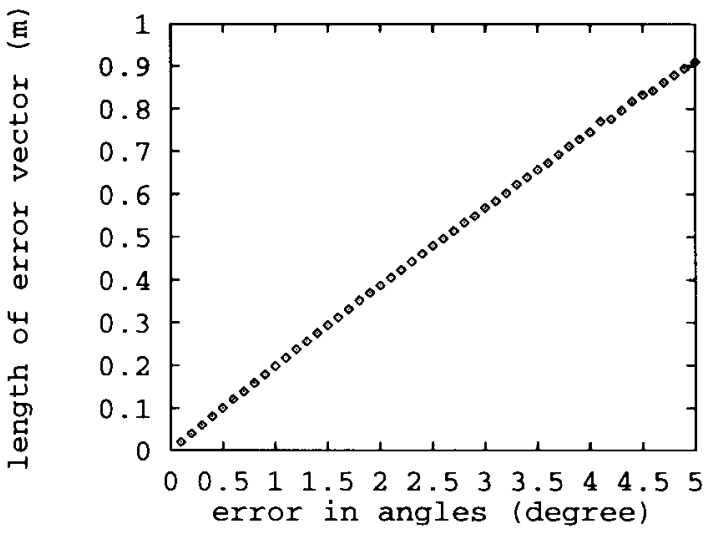

Fig. 9. Simulation results using algorithm Position Estimator on an input of 11 landmarks and angle measurements witherror uniformly distributed over $[-\Delta \varphi, \Delta \varphi]$ where $\Delta \varphi$ ranges from 0 to $5^{\circ}$. The error $\left\|p_{\text {actual }}-p\right\|$ in meters is shown as a function of $\Delta \varphi$ in degrees.

We can now obtain the full expression for $r=$ $\left(A^{T} A\right)^{-1} A^{T} c=\left(n D-b_{x} b_{x}^{T}-b_{y} b_{y}^{T}\right)^{-1} s$ by substituting $K^{-1} s, K^{-1} b_{y}$, and $(n D)^{-1}$ into (9).

If the $i$ th landmark is very close to the robot's position, the least squares method described above may yield a negative $r_{i}$. In this case ratio $r_{i}$ should be positive, but almost zero. Thus, we assign it to be zero in procedure Ratios-Calculator:

Ratios-Calculator $\left(b_{1}, \cdots, b_{n}, s_{1}, \cdots, s_{n}\right)$

1. compute diagonal matrix $(n D)^{-1}$

2. compute vector $K^{-1} s$ using (10)

3. compute vector $K^{-1} b_{y}$ using (11)

4. compute vector $r=\left(r_{i}, \cdots, r_{n}\right)$ using (9)

5. for $i=1$ to $n$

6. if $r_{i}<0$ then $r_{i} \leftarrow 0$

\section{Correctness and Analysis of Position Estimator}

In order to establish the correctness of procedure Position Estimator we first need to prove the following lemmas. We use the notation $D=\operatorname{diag}\left(d_{i}\right)$ to denote a diagonal matrix $D$ whose $i$ th diagonal element is $d_{i}$. Given some matrix $M$ we write $M>0$ if $M$ is positive definite and $M \geq 0$ if $M$ is positive semidefinite.

Lemma 2: Let matrix $D=\operatorname{diag}\left(b_{x i}^{2}+b_{y i}^{2}\right), b_{x}^{T}=$ $\left(b_{x 1}, \cdots, b_{x n}\right)$, and $b_{y}^{T}=\left(b_{y 1}, \cdots, b_{y n}\right)$. Matrix $A^{T} A=$ $2\left(n D-b_{x} b_{x}^{T}-b_{y} b_{y}^{T}\right)$ is nonsingular if and only if the vectors $b_{x}$ and $b_{y}$ are linearly independent.

Proof: Since $A^{T} A \geq 0$, we know that $A^{T} A$ is singular if and only if there exists a vector $z, z \neq 0$ such that $z^{T} A^{T} A z=0$ or equivalently

$$
\begin{aligned}
z^{T}\left(n D-b_{x} b_{x}^{T}-b_{y} b_{y}^{T}\right) z & =0 \\
z^{T} n D z & =z^{T} b_{x} b_{x}^{T} z+z^{T} b_{y} b_{y}^{T} z \\
n z^{T} \operatorname{diag}\left(b_{x i}^{2}+b_{y i}^{2}\right) z & =\left(z^{T} b_{x}\right)\left(b_{x}^{T} z\right)+\left(z^{T} b_{y}\right)\left(b_{y}^{T} z\right) \\
n \sum_{i=1}^{n}\left(b_{x i}^{2}+b_{y i}^{2}\right) z_{i}^{2} & =\left(\sum_{i=1}^{n} b_{x i} z_{i}\right)^{2}+\left(\sum_{i=1}^{n} b_{y i} z_{i}\right)^{2} .
\end{aligned}
$$

Note that for any numbers $a_{1}, \cdots, a_{n}$ we know that $\left(\sum_{i=1}^{n} a_{i}\right)^{2} \leq n \sum_{i=1}^{n} a_{i}^{2}$ and the equality holds if and only if $a_{i}=a_{j}$ for all indexes $i$ and $j$. Therefore, $A^{T} A$ is singular 


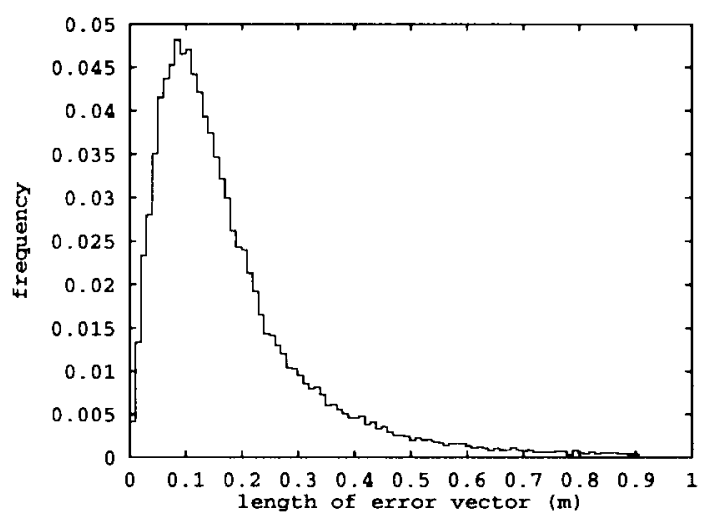

(a)

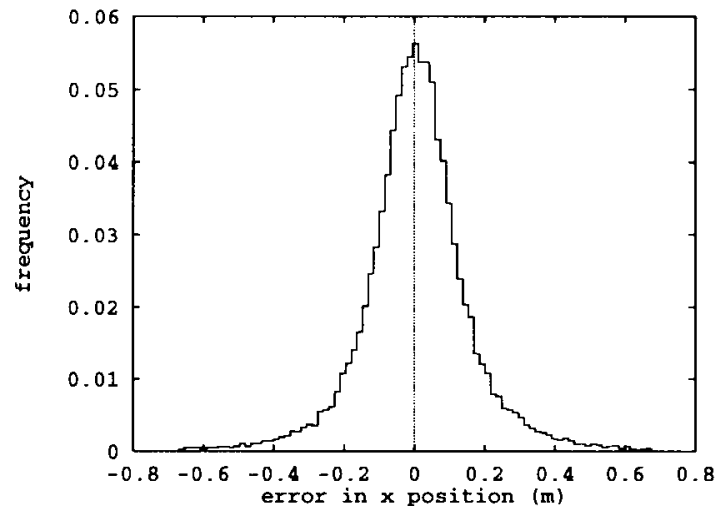

(c)

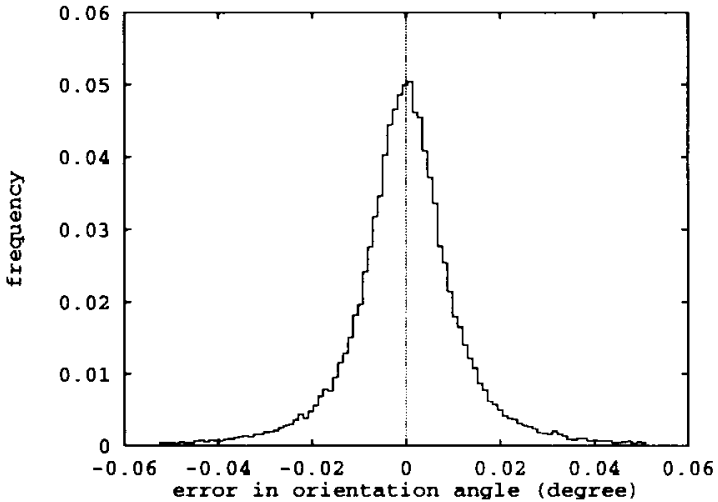

(b)

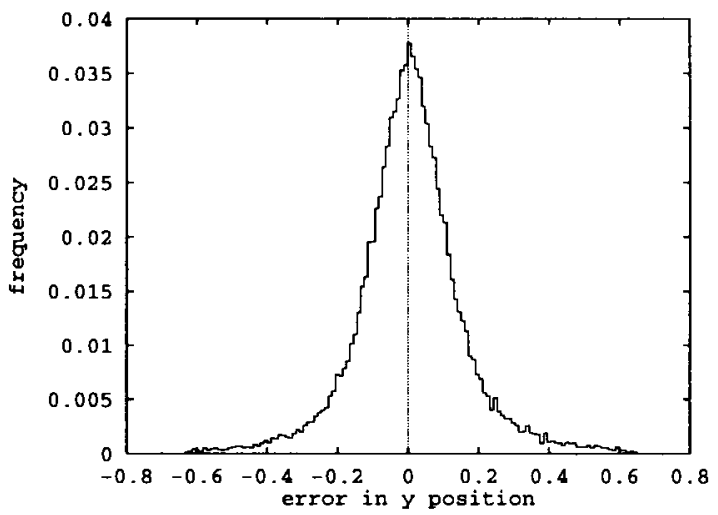

(d)

Fig. 10. Distribution of errors for $\Delta \varphi=1^{\circ}$. (a) The distribution of the length of error vector $\left\|p_{\text {actual }}-p\right\|$ with mean 19.7 cm and standard deviation $23.9 \mathrm{~cm}$. (b) The distribution of the error in orientation $\left|\theta_{\text {actual }}-\theta\right|$ with almost zero mean and standard deviation $0.017^{\circ}$. (c) The distribution of error in $x$ position $\mid p_{x}$, actual $-p_{x} \mid$ with mean $0.93 \mathrm{~cm}$ and standard deviation $0.22 \mathrm{~cm}$. (d) The distribution of error in $y$ position $\mid p_{y}$, actual $-p_{y} \mid$ with mean $10.6 \mathrm{~cm}$ and standard deviation $21.4 \mathrm{~cm}$.

if and only if

$$
b_{x i} z_{i}=b_{x j} z_{j} \quad \text { and } \quad b_{y i} z_{i}=b_{y j} z_{j}
$$

for all indexes $i$ and $j$. This means that $b_{x}=\alpha z$ and $b_{y}=\beta z$ for some constants $\alpha$ and $\beta$. Thus we get that $b_{x}=(\alpha / \beta) b_{y}$ which means that $b_{x}$ and $b_{y}$ are linearly dependent.

The following lemma gives the geometrical interpretation of Lemma 2.

Lemma 3: Matrix $A^{T} A=2\left(n D-b_{x} b_{x}^{T}-b_{y} b_{y}^{T}\right)$ is singular if and only if the landmarks and the robot's position are arranged in a perfect circle or on a line.

Proof: According to Lemma 2 we have to find a geometric criterion for vectors $b_{x}$ and $b_{y}$ to be linearly dependent. The rank of the $n \times 2$ matrix $b_{x} b_{y}$ whose two columns consist of vectors $b_{x}$ and $b_{y}$ is one if vectors $b_{x}$ and $b_{y}$ are collinear. Note that

$$
\operatorname{rank}\left(b_{x} b_{y}\right)=\operatorname{rank}\left(\begin{array}{c}
b_{x}^{T} \\
b_{y}^{T}
\end{array}\right)
$$

where $\left(\begin{array}{l}b_{x}^{T} \\ b_{y}^{T}\end{array}\right)$ is the matrix whose rows are vectors $b_{x}^{T}$ and $b_{y}^{T}$. By definition (see Section V-B) we have

$$
\left(\begin{array}{l}
b_{x}^{T} \\
b_{y}^{T}
\end{array}\right)=\left(\begin{array}{l}
b_{x 1} \cdots b_{x n} \\
b_{y 1} \cdots b_{y n}
\end{array}\right)=\left(b_{1} \cdots b_{n}\right)
$$

since $b_{k}=\left(b_{x k}, b_{y k}\right)^{T}$, for $1 \leq k \leq n$. Therefore, it is sufficient for the proof to find the geometrical interpretation of the collinearity of the 2-D vectors $b_{k}$, for $1 \leq k \leq n$. That is, we need a geometrical interpretation for the existence of a complex number $\eta$ such that $b_{k}=\alpha_{k} \eta$, where $\alpha_{k}$ is a real number.

We also have

$$
b_{k}=c_{k} e^{j \varphi_{k}}=\frac{e^{j \varphi_{k}}}{v_{k}}=\frac{e^{j \varphi_{k}}}{z_{k}-z_{0}}
$$

where vector $v_{k}$ connects landmark $z_{0}$ with the landmarks $z_{k}$ and $\varphi_{k}$ is the visual angle between $z_{0}$ and $z_{k}$ from the robot's position for $1 \leq k \leq n$ as illustrated in Fig. 7. (Note that $b_{k}=\left(b_{x k}, b_{y k}\right)^{T}$ is not a vector in the robot-centered coordinate system.)

Without loss of generality, we use a robot-centered coordinate system in which the $x$-axis is aligned with the vector to landmark $z_{0}$ (see Fig. 7). The landmarks in this coordinate system are defined as $z_{k}=\left|z_{k}\right| e^{j \varphi_{k}}$ for $0<k \leq n$. Thus, $b_{k}$ can also be expressed as

$$
b_{k}=\frac{z_{k}}{z_{k}-z_{0}} \frac{1}{\left|z_{k}\right|}=\frac{z_{k}}{z_{k}-z_{0}} k
$$

where $k$ is defined to be $1 /\left|z_{k}\right|$. Then vectors $b_{k}, 1 \leq k \leq b_{n}$, are linearly independent if for all indexes $k$

$$
b_{k}=\frac{z_{k}}{z_{k}-z_{0}} k=\alpha_{k} \eta
$$

for some fixed complex number $\eta$ and real $\alpha_{k}$. We can also 


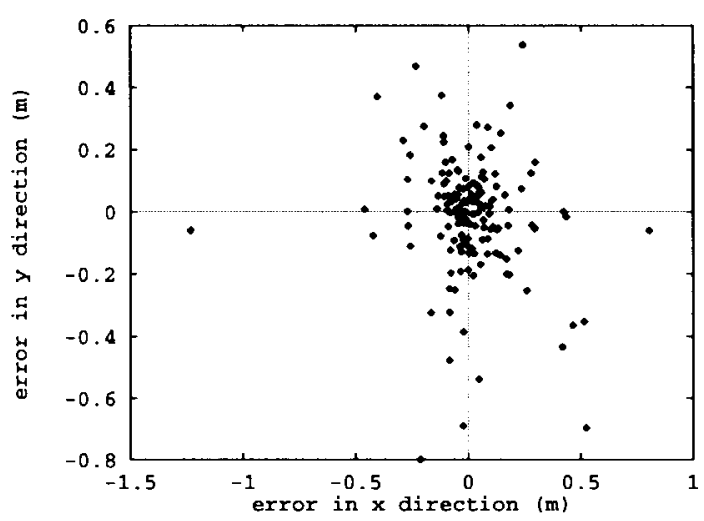

(a)

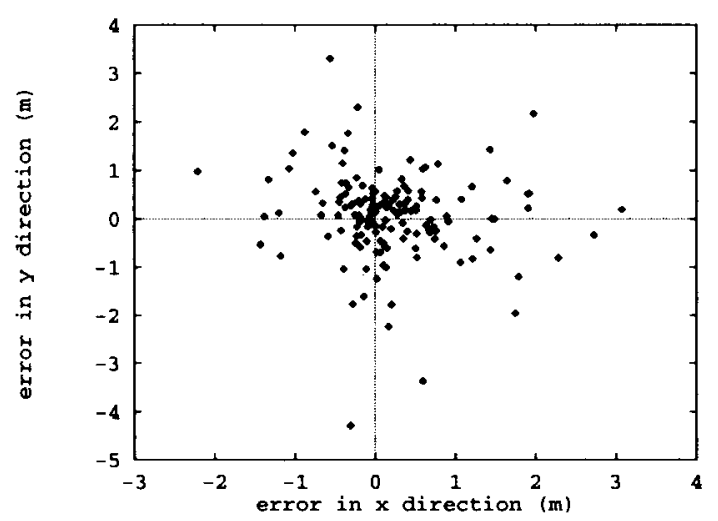

(b)

Fig. 11. Computed positions of the robot in the two-dimensional environment from 200 sets of 11 landmarks for $\Delta \varphi=1^{\circ}$ (a). (b) $\Delta \varphi=5^{\circ}$.

write this as

$$
\frac{z_{k}}{z_{k}-z_{0}}=\beta_{k} \eta
$$

for $\beta_{k}=\alpha_{k} / k$ which is equivalent to $\beta_{k} \eta z_{0}=\beta_{k} \eta z_{k}-z_{k}$ and

$$
\frac{\beta_{k} \eta z_{0}}{\beta_{k} \eta-1}=z_{k}
$$

for $\beta_{k} \eta-1 \neq 0$. We can view this as a mapping $f$ of points $w=\beta_{k} \eta$ in the complex $w$-plane into the complex $z$-plane where each landmark $z_{k}$ is a point in the $z$-plane

$$
f(w)=\frac{z_{0} w}{w-1}=z
$$

This mapping is called a Möbius transformation (see for example [32]). The Möbius transformation maps the line $w=\beta \eta$ in the $w$-plane into a circle in the $z$-plane. Since $f(0)=0$, the circle passes through the origin of the $z$-plane. Thus, landmarks $z_{1}, \cdots, z_{n}$ and the robot at $(0,0)$ lie on a circle if the vectors $b_{k}, 1 \leq k \leq b_{n}$, are linearly independent. Landmark $z_{0}$ lies on the same circle since

$$
\lim _{\beta \rightarrow \infty} \frac{\beta \eta z_{0}}{\beta \eta-1}=\lim _{\beta \rightarrow \infty} \frac{\eta z_{0}}{\eta-1 / \beta}=z_{0} .
$$

Note that we assumed $\beta_{k} \eta-1 \neq 0$. If $\beta_{k} \eta=1$, then we know that $b_{k}=\frac{e^{j \psi_{k}}}{z_{k}-z_{0}}=k \beta_{k} \eta=k$ which means that $\varphi_{k}=0$. In this case it follows that all landmarks $z_{0}, \cdots, z_{n}$ lie on the real axis of the $z$-plane. Thus, the landmarks and the robot lie on one line.

The properties of a Möbius transformation also hold for inverse transforms. Therefore, given landmarks arranged on a circle or on a line, it follows that vectors $b_{k}, 1 \leq k \leq b_{n}$, are linearly independent and matrix $A^{T} A$ is singular.

The singularity condition is easily tested through some preprocessing that makes sure that the landmarks are not all arranged in a circle or on a line when procedure Position Estimator is called. In practice it is highly unlikely that the landmarks all lie on a perfect circle or line including the robot's position.

Lemma 4: If landmarks are correctly identified and there is no noise in the measurements of the visual angles between the landmarks, then procedure Position Estimator returns the actual position of the robot.

Proof: Let $\hat{z}_{i}^{(r)}=\hat{l}_{i} e^{j \hat{\tau}}$ be the vector to the $i$ th landmark in the robot coordinate system that is oriented as the external

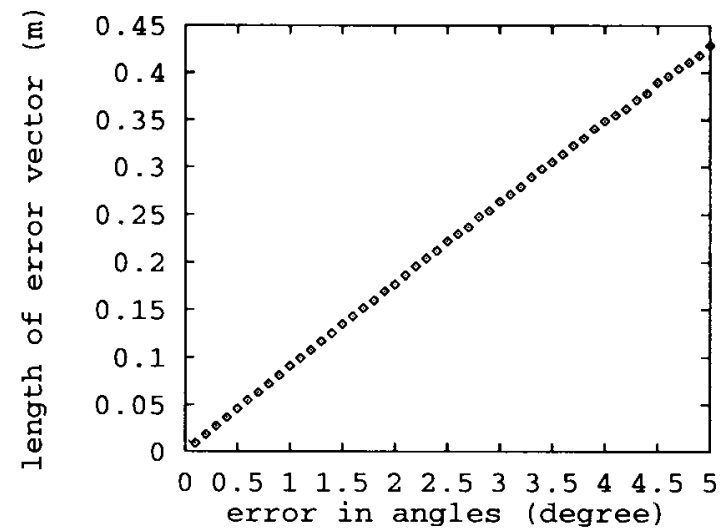

Fig. 12. Simulation results using algorithm Position Estimator on an input of 11 landmarks surrounding the robot and angle measurements with error uniformly distributed over $[-\Delta \varphi, \Delta \varphi]$ where $\Delta \varphi$ ranges from 0 to $5^{\circ}$. The error $\left\|p_{\text {actual }}-p\right\|$ in meters is shown as a function of measurement errors in degrees.

coordinate system. Note that the actual position of the robot is at $p_{\text {actual }}=z_{0}^{(e)}-\hat{z}_{0}^{(r)}$. The algorithm calculates $p=$ $z_{0}^{(e)}-\frac{1}{n} \sum_{i=1}^{n} \hat{z}_{0, i}^{(r)}$ which is equivalent to

$$
\begin{aligned}
p & =z_{0}^{(e)}-\frac{1}{n} \sum_{i=1}^{n} \frac{1}{r_{i} b_{i}-c_{i}} \\
& =z_{0}^{(e)}-\frac{1}{n} \sum_{i=1}^{n} \frac{1}{\hat{l}_{i}} \frac{1}{\hat{l}_{0} e^{j \varphi_{i}}-\frac{1}{\hat{v}_{i}}} \\
& =z_{0}^{(e)}-\frac{1}{n} \sum_{i=1}^{n} \frac{\hat{l}_{0} e^{j \hat{\tau}_{0}}\left(\hat{z}_{i}^{r}-\hat{z}_{0}^{(r)}\right)}{\hat{l}_{i} e^{j \hat{\tau}_{i}}-\hat{l}_{0} e^{j \hat{\tau}_{0}}} \\
& =z_{0}^{(e)}-\frac{1}{n} \sum_{i=1}^{n} \frac{\hat{z}_{0}^{(r)}\left(\hat{z}_{i}^{(r)}-\hat{z}_{0}^{(r)}\right)}{\hat{z}_{i}^{(r)}-\hat{z}_{0}^{(r)}} \\
& =z_{0}^{(e)}-\frac{1}{n} n \hat{z}_{0}^{(r)} \\
& =z_{0}^{(e)}-\hat{z}_{0}^{(r)} .
\end{aligned}
$$

Thus, the algorithm calculates $p=p_{\text {actual }}$.

Lemmas 2 and 3 establish the correctness of our algorithm:

Theorem 1: Given a set of noisy measurements of landmarks which are not all arranged in a circle or line, algorithm Position Estimator that determines the position of the robot 


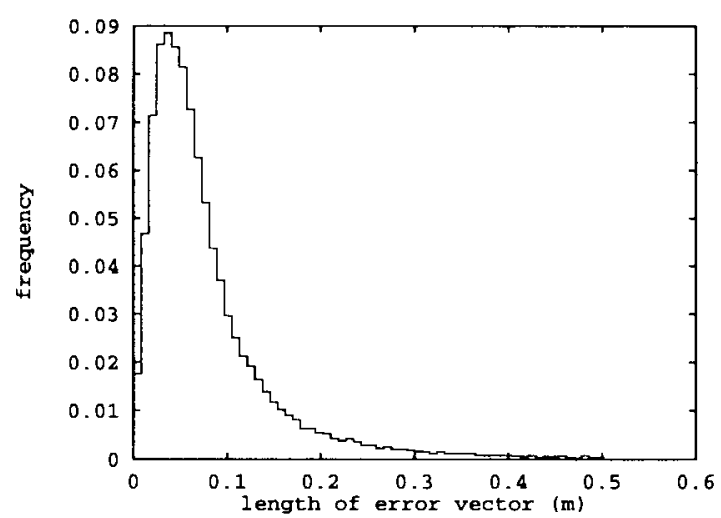

(a)

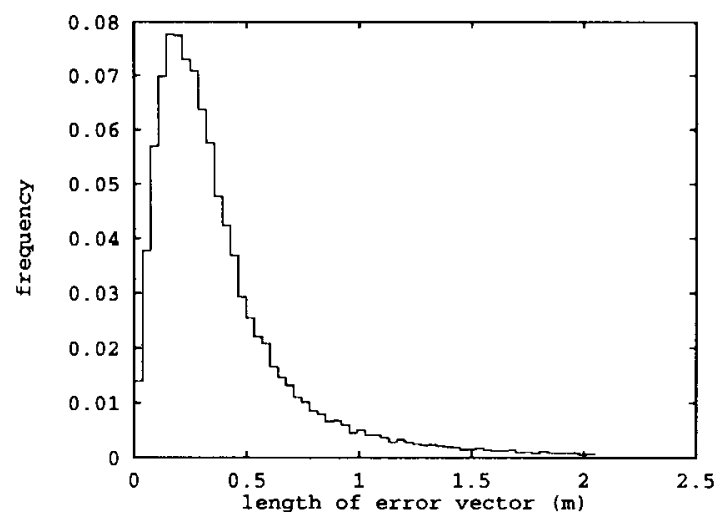

(c)

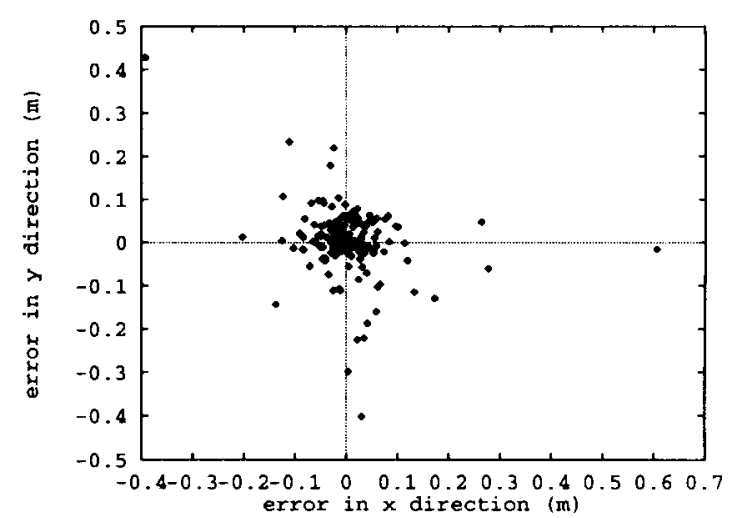

(b)

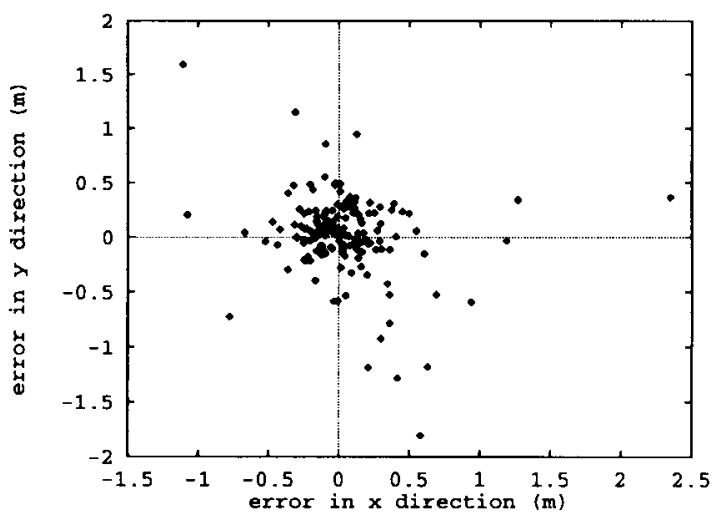

(d)

Fig. 13. Experiments in which the robot is surrounded by 11 landmarks. On the left, the distributions of the length of error vectors $\left\|p_{\text {actual }}-p\right\|$ for $\Delta \varphi$ uniformly distributed within $[-1,1]$ and $[-5,5]$. On the right, Computed positions of the robot in the two-dimensional environment for $\Delta \varphi=1$ and $5^{\circ}$.

such that the square of errors in the equations $A r=c$ is minimized.

Theorem 2: The algorithm Position Estimator runs in time linear in the number of landmarks.

Proof: Due to the special form of vector $s=\frac{1}{2} A^{T} c$ it can be calculated in time linear in $n$ by first computing $\sum_{j=1}^{n} c_{j x}$ and $\sum_{j=1}^{n} c_{j x}$ and then computing each entry $s_{i}$ of vector $s$ following (8).

Algorithm Position Estimator calls procedure RatiosCalculator which calculates vector $r$ by substituting matrix $(n D)^{-1}$ and vectors $K^{-1} s$ and $K^{-1} b_{y}$ into (9). Matrix $(n D)^{-1}$ can be computed in $O(n)$ time if we represent this diagonal matrix by a vector of its diagonal entries. The $i$ th diagonal entry of $(n D)^{-1}$ is $1 /\left(n\left(b_{x i}^{2}+b_{y i}^{2}\right)\right)$. Using this vector representation means that multiplying matrix $(n D)^{-1}$ with vectors $s$ and $b_{x}$ can also be done in linear time. Dot products $\left((n D)^{-1} b_{x}\right)^{T} s$ and $\left((n D)^{-1} b_{x}\right)^{T} b_{x}$ take $O(n)$ time to compute. Given vectors $(n D)^{-1} s$ and $(n D)^{-1} b_{x}$ and scalars $\left((n D)^{-1} b_{x}\right)^{T} s$ and $\left((n D)^{-1} b_{x}\right)^{T} b_{x}$, the computation of (10) can be concluded in $O(1)$ time.

With a similar argument we can show that (11) and (9) can be computed in linear time.

\section{Quality of Position Estimator}

This section reports results of simulation experiments using procedure Position Estimate. In the experiments, the actual robot position $p_{\text {actual }}=\left(p_{x, \text { actual }}, p_{y, \text { actual }}\right)$ is compared to the position $p=\left(p_{x}, p_{y}\right)$ that is computed by procedure Position Estimate. The actual robot orientation $\theta_{\text {actual }}$ is compared to the orientation $\theta$ that is also computed by procedure Position Estimate.

In the experiments, the errors $\left|p_{x, \text { actual }}-p_{x}\right|$ and $\mid p_{y, \text { actual }}-$ $p_{y} \mid$ in the $x$ - and $y$-coordinates of the position are computed. The length of the error vector $\left\|p_{\text {actual }}-p\right\|$ and the orientation error $\left|\theta_{\text {actual }}-\theta\right|$ are also computed.

Without loss of generality, the actual robot position $p_{\text {actual }}$ is assumed to be at the origin of the map, i.e., $p_{\text {actual }}=(0,0)$ and the robot is assumed to be oriented in the direction of the $x$-axis, i.e., $\theta_{\text {actual }}=0^{\circ}$.

First we randomly place 11 landmarks in a $10 \mathrm{~m} \times 10 \mathrm{~m}$ area using a uniform distribution where the robot is at a corner of this area. Note that this scenario uses only a quarter of the robot's surrounding area. It assumes that the robot cannot get omnidirectional input as Ratbot can, but instead has a conventional camera setup.

In an attempt to simulate worst-case measurement errors, we first add a constant fraction $\Delta \varphi$ of a degree to each angle. Fig. 8 shows results of experiments in which the added noise $\Delta \varphi$ ranges from 0 to $5^{\circ}$. We average our results over 100000 scenarios with 11 landmarks, distributed uniformly in the 10 by 10 meter area. The length of the error vector $p_{\text {actual }}-p$ is $57 \mathrm{~cm}$ for $1^{\circ}$ noise, $1.1 \mathrm{~m}$ for $2^{\circ}$ noise, and $2.57 \mathrm{~m}$ for $5^{\circ}$ for noise in every angle. The error in the position angle $\theta$ is 
$2^{\circ}$ for $1^{\circ}$ angle noise, $4.2^{\circ}$ for $2^{\circ}$ angle noise, and $9.8^{\circ}$ for $5^{\circ}$ angle noise.

Next we show results of experiments in which the angle errors are not fixed, but uniformly distributed between $[-\Delta \varphi, \Delta \varphi]$ where $\Delta \varphi$ also ranges from 0 to $5^{\circ}$. Figs. 9 and 10 show the results of the experiments with 40000 different environments that include 11 landmarks. Fig. 9 shows the length of the error vector as a function of the visual angle error. The length of the error vector $p_{\text {actual }}-p$ is $19 \mathrm{~cm}$ for angle noise randomly chosen within $\left[-1^{\circ}, 1^{\circ}\right], 38 \mathrm{~cm}$ for angle noise randomly chosen within $\left[-2^{\circ}, 2^{\circ}\right]$, and $90 \mathrm{~cm}$ for angle noise randomly chosen within $\left[-5^{\circ}, 5^{\circ}\right]$. The error in position angle $\theta$ is on average zero.

Fig. 10 shows how the errors, ||$p_{\text {actual }}-p||,\left|p_{x, \text { actual }}-p_{x}\right|$, $\left|p_{y, \text { actual }}-p_{y}\right|$, and $\left|\theta_{\text {actual }}-\theta\right|$ are distributed for $\Delta \varphi=$ $1^{\circ}$. Fig. 11 shows 200 positions of the robot in the twodimensional environment computed by Position Estimator with input error $\Delta \varphi=1^{\circ}$ and $\Delta \varphi=5^{\circ}$. Note that the distributions for position errors $\left|p_{x, \text { actual }}-p_{x}\right|$ and $\left|p_{y, \text { actual }}-p_{y}\right|$ are almost normal.

In the next set of experiments, the robot is in the center of a $20 \mathrm{~m} \times 20 \mathrm{~m}$ area with eleven landmarks. We expect better results for this scenario in which the robot can take advantage of information provided by all of its surrounding. Indeed, our results show that the error in position has length $8.9 \mathrm{~cm}$ for $\Delta \varphi$ uniformly distributed within [-1,1], length $17.6 \mathrm{~cm}$ for $\Delta \varphi$ uniformly distributed within [-2,2], and length $42.6 \mathrm{~cm}$ for $\Delta \varphi$ uniformly distributed within $[-5,5]$. The results of the experiments are summarized in Figs. 12 and 13.

Note that procedure Position Estimator computes $n$ position estimates $p_{1}, \cdots, p_{n}$ and averages them to obtain estimate $p$. Among $p_{1}, \cdots, p_{n}$, there may be some outliers that are not very close to the final position estimate $p$. However, in our experiments we observed that there is a one-to-one correspondence between each landmark $z_{i}$ and estimate $p_{i}$. Each outlier $p_{i}$ corresponds to either a misidentified landmark $z_{i}$ or a noisy angle measurement for landmark $z_{i}$. Fig. 14 illustrates this behavior of procedure Position Estimator. Given an input of 21 landmarks $z_{0}, \cdots, z_{20}$, the procedure computes 20 position estimates $p_{1}, \cdots, p_{20}$. To model outlier errors resulting either from two noisy angle measurements or from two misidentified landmarks, we add a fixed error of $20^{\circ}$ to two arbitrary angles. The other angles have an additive error uniformly distributed within $\left[-1^{\circ}, 1^{\circ}\right]$.

Our experiments show that the outlier landmarks produce position estimates that are far from the actual position of the robot compared to the other estimates. So instead of taking the centroid of all position estimates $p_{1}, \cdots, p_{20}$ in line 12 of Position Estimator, we can obtain better results if we modify the algorithm such that we discard the outlier position estimates and calculate the centroid of the remaining points.

However, it is even better to call Position Estimator on an input of 19 landmarks (without the outlier landmarks). Fig. 14 shows the results of our computation on two scenarios in which the length of the error vector is computed. For each of the scenarios, we show position estimates that are computed with and without the outlier landmarks. In the first scenario,

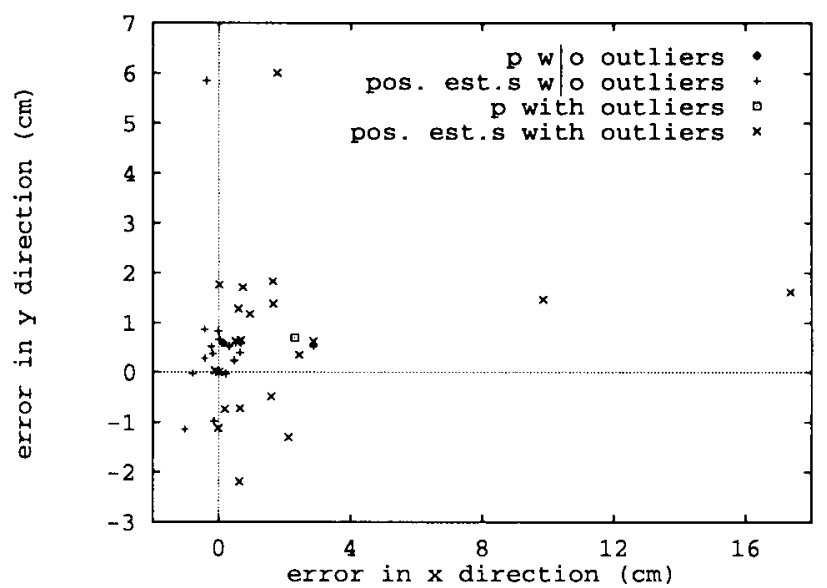

(a)

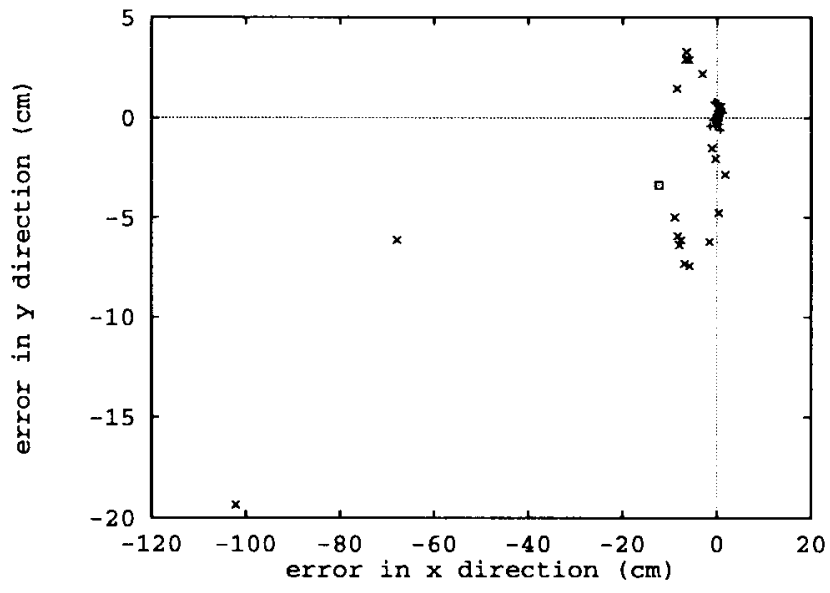

(b)

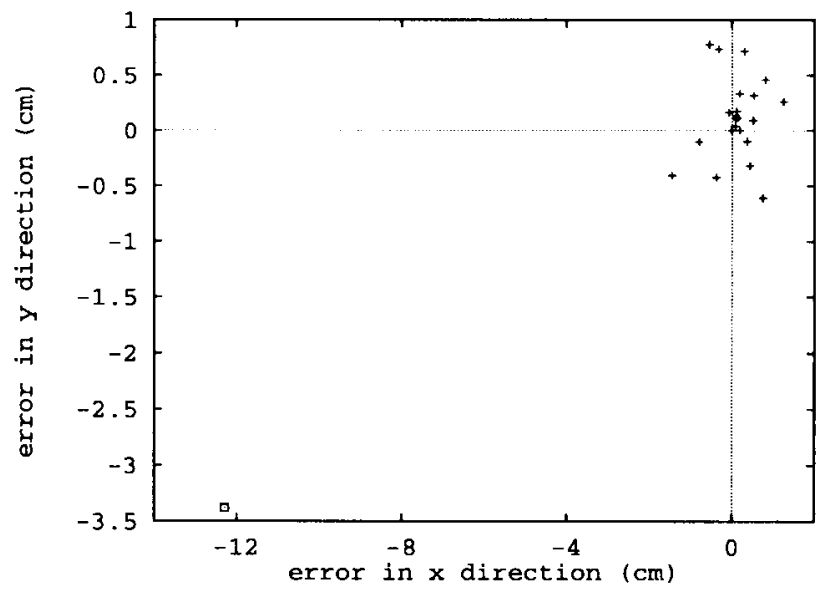

(c)

Fig. 14. Two experiments with 21 landmarks including two outliers. In the first experiment (a), position estimate $p$ computed with outliers is a $(2.3 \mathrm{~cm}$, $0.7 \mathrm{~cm})$ and $p$ computed without outliers is at $(0.1 \mathrm{~cm}, 0.6 \mathrm{~cm})$. In the second experiment (b) and (c), position estimate $p$ computed with outliers is at $(-12.3$ $\mathrm{cm},-3.4 \mathrm{~cm})$ and $p$ computedd without outliers is at $(0.1 \mathrm{~cm}, 0.1 \mathrm{~cm})$.

the length of the error vector is $24.0 \mathrm{~cm}$ when computed including outlier landmarks, and $6.0 \mathrm{~cm}$ when computed without the outlier landmarks. In the second scenario, the length of the error vector is $127 \mathrm{~cm}$ when computed with outlier landmarks, and $1.6 \mathrm{~cm}$ when computed without the outlier landmarks. 


\section{CONCLUSION}

We described an algorithm for estimating the position and orientation of a mobile robot given noisy input data. The key advantages of our Position Estimator over the approach described in Section IV is that 1) the algorithm runs in time linear in the number of landmarks, 2) the algorithm provides a position estimate which is very close to the actual robot position and orientation, and 3) large errors in some measurements (i.e., outliers) can be found.

Our algorithm does not use information about the motion of the mobile robot: the history of position estimates, the commands that make the robot move, and the uncertainties in these commands. Procedure Position Estimator could be incorporated into a navigation algorithm that keeps track of this information, for example, by using Kalman filtering.

\section{REFERENCES}

[1] S. Judd, private communication, 1993.

[2] T. R. Hancock and S. J. Judd, "Hallway navigation using simple visual correspondence algorithms," Tech. Rep. SCR-94-479, Siemens Corporate Res., Princeton, NJ, 1993.

[3] R. Greiner and R. Isukapalli, "Learning useful landmarks," Tech. Rep., Siemens Corporate Res., Princeton, NJ, 1993.

[4] K. Sugihara, "Some location problems for robot navigation using a single camera," Comput. Vision, Graphics, and Image Processing, vol. 42, pp. 112-129, 1988.

[5] D. Avis and H. Imai, "Locating a robot with angle measurements," $J$. Symbolic Computat., vol. 10, pp. 311-326, 1990.

[6] E. Krotov, "Mobile robot localization using a single image," in Proc. IEEE Int. Conf. Robot. Automat., Scottsdale, AZ, May 1989, vol. 2, pp. 978-983.

[7] K. T. Sutherland and W. B. Thompson, "Inexact navigation," in Proc. 1993 IEEE Int. Conf. Robot. Automat., Atlanta, GA, May 1993, vol. 1, pp. $1-7$.

[8] R. A. Brooks, "Aspects of mobile robot visual map making," in Robotics Research, H. Hanafusa and H. Inoue, Eds. Cambridge, MA: MIT Press, 1985.

[9] M. J. Mataric, "A distributed model for mobile robot environmentlearning and navigation," Tech. Rep. AI-TR-1228, M.I.T., Cambridge, MA, 1990.

[10] A. Elfes, "A sonar-based mapping and navigation system," IEEE $J$ Robot. Automat., vol. RA-3, pp. 249-265, June 1987.

[11] T. S. Levitt, D. T. Lawton, D. M. Chelberg, and P. C. Nelson, "Qualitative navigation," in Proc. DARPA Image Understanding Workshop, Los Angeles, CA, Feb. 1987, vol. 2, pp. 447-465.

[12] T. S. Levitt, D. T. Lawton, D. M. Chelberg, K. V. Koitzsch, and J. W. Dye, "Qualitative navigation II," in Proc. DARPA Image Understanding Workshop, Cambridge, MA, 1988, pp. 319-326.

[13] B. J. Kuipers and T. S. Levitt, "Navigation and mapping in large-scale space," AI Mag., vol. 9, no. 2, pp. 25-43, 1988.

[14] R. Chatila and J.-P. Laumond, "Position referencing and consistent world modeling for mobile robots," in Proc. 1985 IEEE Int. Conf. Robot. Automat., St. Louis, MO, Mar. 1985, pp. 138-145.

[15] R. C. Smith and P. Cheeseman, "On the representation and estimation of spatial uncertainty," Int. J. Robot. Res., vol. 5, no. 4, pp. 56-68, 1986.

[16] A. Kosaka and A. Kak, "Fast vision-guided mobile robot navigation using model-based reasoning and reduction of uncertainties," Computer Vision, Graphics, and Image Processing: Image Understanding, vol. 56, no. 3, pp. 271-329, 1992.

[17] J. Crowley, "World modeling and position estimation for a mobile robot using ultrasonic ranging," in Proc. 1989 IEEE Int. Conf. Robot. Automat., Scottsdale, AZ, May 1989, vol. 2, pp. 674-680.

[18] J. J. Leonard and H. F. Durrant-Whyte, "Simultaneous map building and localization for an autonomous mobile robot," in Proc. IEEE Int.
Conf. Intelligent Robot Syst., Osaka, Japan, Nov. 1991.

[19] Y. Watanabe and S. Yuta, "Position estimation of mobile robots with internal and external sensors using uncertainty evolution technique," in Proc. 1990 IEEE Int. Conf. Robot. Automat., Cincinnati, OH, May 1990, pp. 2011-2016.

[20] P. Burlina, D. DeMenthon, and L. S. Davis, "Navigation with uncertainty: I. Reaching a goal in a high collision risk region," Tech. Rep. CAR-TR-565, CS-TR-2717, Univ. Maryland, College Park, June 1991.

[21] L. Matthies and S. A. Shafer, "Error modeling in stereo navigation," Tech. Rep. CMU/CS/86-140, Carnegie-Mellon Univ., Pittsburgh, PA, 1986.

[22] M. Drumheller, "Mobile robot localization using sonar," IEEE Trans. Pattern Anal. Machine Intell., vol. PAMI-9, pp. 325-332, Mar. 1987. Also published as MIT AI-Memo 826, Jan. 1985.

[23] F. Chenavier and J. L. Crowley, "Position estimation for a mobile robot using vision and odometry," in Proc. 1992 IEEE Int. Conf. Robot. Automat., Nice, France, 1992, pp. 2588-2593.

[24] S. Cooper and H. Durrant-Whyte, "A Kalman filter model for GPS navigation of land vehicles," in Proc. IEEE/RSJ/GI Int. Conf. Intell. Robots Syst., Munich, Germany, Sept. 1994, pp. 157-163.

[25] L. Kleeman, "Optimal estimation of position and heading for mobile robots using ultrasonic beacons and dead-reckoning," in Proc. 1992 IEEE Int. Conf. Robot. Automat., Nice, France, 1992, pp. 2582-2587.

[26] B. K. P. Horn, Robot Vision. Cambridge, MA and New York: MIT Elect. Eng. Comput. Sci. Series, The MIT Press/McGraw-Hill, 1986.

[27] J. J. Craig, Introduction to Robotics: Mechanics and Control. Reading, MA: Addison-Wesley, 1989

[28] Gellert, Küster, Hellwich, and Kästner (Eds.), Mathematik, VEB Verlag Enzykopädie, Leipzig, Germany, 1967.

[29] G. Strang, Linear Algebra and Its Applications. New York: Academic, 1976.

[30] A. Albert, Regression and the Moore-Penrose Pseudoinverse. New York: Academic, 1972

[31] L. Gurvits and M. Betke, Robot Navigation Using Landmarks. In preparation.

[32] R. V. Churchill, Introduction to Complex Variables and Applications. New York: McGraw-Hill, 1948

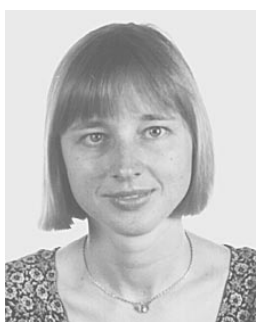

Margrit Betke received the S.M. and Ph.D. degrees in computer science and electrical engineering from the Massachusetts Institute of Technology in 1992 and 1995 , respectively.

She joined the University of Maryland Institute for Advanced Computer Studies in 1995. Her research interests are in computer vision, robotics, and machine learning. Her previous work has spanned a range of topics including real-time object recognition and tracking, mobile robot navigation, and autonomous exploration of unknown environments. She is also interested in the application of computer vision to intelligent vehicles.

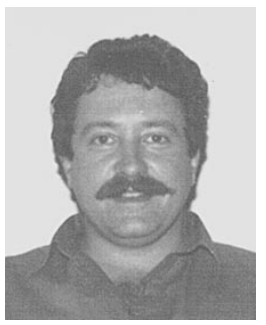

Leonid Gurvits was born in Chernovtsy, Ukraine (formerly, USSR). He received the M.S. degree from Chernovtsy State University and the Ph.D. degree from Gorky State University, both in mathematics.

$\mathrm{He}$ is an Associate Editor of Mathematics of Control, Signals, and Systems. His areas of interes are linear and nonlinear control, linear algebra, functional analysis, Markov chains, statistics, combinatorics (linear and multilinear algebra), approximation theory, and learning (TD-methods). He has published more than 40 articles. 